\title{
Discrimination of Paroxysmal and Persistent Atrial Fibrillation in Patients With New-Onset Atrial Fibrillation
}

\author{
Tazuru Igarashi, ${ }^{1}$ MD, Shinichi Niwano, ${ }^{1}$ MD, Hidehira Fukaya, ${ }^{1}$ MD, \\ Tomoharu Yoshizawa, ${ }^{1}$ MD, Hironori NaKamura, ${ }^{1}$ MD, Tamami FujiIshi, ${ }^{1}$ MD, \\ Naruya Ishizue, ${ }^{1} \mathrm{MD}$, Jun OiKawa, ${ }^{1} \mathrm{MD}$, Jun Kishihara, ${ }^{1} \mathrm{MD}$, \\ Masami Murakami, ${ }^{1} \mathrm{MD}$, Hiroe Niwano, ${ }^{1} \mathrm{MD}$, and Junya AKo, ${ }^{1}$ MD
}

\section{SUMMARY}

Discrimination between paroxysmal and persistent atrial fibrillation (PAF and persistent AF) is important for determining the therapeutic strategy in patients with new-onset AF. We evaluated various clinical factors and $\mathrm{P}$ wave morphology to discriminate $\mathrm{PAF}$ and persistent $\mathrm{AF}$ patients in patients with new-onset $\mathrm{AF}$.

The study population consisted of 79 patients with new-onset $\mathrm{AF}$ ( $70.3 \pm 10.8$ years, female:male $33: 46)$ who were retrospectively selected from 8,632 AF patients in the Kitasato University Hospital ECG storing system. PAF $(n=38)$ and persistent $\mathrm{AF}(n=41)$ patients were diagnosed by whether the initial PAF episode continued for 1 week. The P wave morphologies were analyzed using the most recent 12 lead-ECG recording of sinus rhythm. P wave dispersion was defined as the difference between the maximum and minimum durations of all leads. Along with these data, various clinical factors were evaluated and compared between $\mathrm{PAF}$ and persistent $\mathrm{AF}$ patients.

Multivariate analysis identified $\mathrm{P}$ wave dispersion $(56.6 \pm 14.8$ versus $66.5 \pm 12.8 \mathrm{msec}, P=0.002)$ and left atrial dimension (LAD: $40.2 \pm 7.0$ versus $47.7 \pm 8.2 \mathrm{~mm}, P<0.001$ ) as independent factors for discrimination between PAF and persistent AF patients. Combining these two parameters achieved a specificity of $88.9 \%$, a positive predictive value of $81.8 \%$, a sensitivity of $95.3 \%$, and a negative predictive value of $88.9 \%$.

In patients with new-onset $\mathrm{AF}, \mathrm{P}$ wave dispersion and $\mathrm{LAD}$ were independent factors for discrimination between PAF and persistent AF. (Int Heart J 2016; 57: 573-579)

Key words: $\mathrm{P}$ wave

$\mathrm{I}^{\mathrm{T}}$ clinical practice, identification of the clinical stage of atrial fibrillation (AF) is an important issue to enable clinicians to determine a therapeutic strategy, especially in patients with new-onset AF, in whom it is important to determine the indication of antiarrhythmic agents or catheter ablation. ${ }^{1-3)}$ When considering catheter ablation for $\mathrm{AF}$, it is important to identify the early stage of AF, ie, paroxysmal atrial fibrillation (PAF), because the efficacy of catheter ablation is higher in PAF patients, and the ablation procedure itself can be simplified. ${ }^{4,5}$ Recently, we have reported that $\mathrm{P}$ wave analysis in the 12-lead ECG of sinus rhythm is useful in determining the emergence of AF in clinical cases. ${ }^{6}$ We have shown that the $\mathrm{P}$ wave amplitude in lead II or V1 was higher, and $\mathrm{P}$ wave dispersion, ie, the dispersion of $\mathrm{P}$ wave duration, was larger in patients with new-onset AF compared with control patients without $\mathrm{AF}^{6}{ }^{6}$ Therefore, we hypothesized that the clinical stage of AF may also be identified by $\mathrm{P}$ wave analysis, as well as with the other clinical parameters. In the present study, we retrospectively identified patients with new-onset $\mathrm{AF}$ in the digital ECG profiling system of our hospital. Patients were classified into PAF and persistent AF by their clinical course in accord- ance with the definitions in the AHA/ACC/ESC guidelines and JCS guidelines. ${ }^{7.8)} \mathrm{P}$ waves in the preceding sinus rhythm state were analyzed as the precursor state for new-onset AF. P wave data together with other clinical parameters were compared between patients with PAF and persistent $\mathrm{AF}$ to determine the clinical factors allowing discrimination between PAF and persistent $\mathrm{AF}$.

\section{Methods}

Study population: Between 2008 and 2012, a total of 106,921 12-lead ECG recordings were stored in the digital ECG profiling system of Kitasato University Hospital. Among these recordings, 8,632 patients exhibited AF. For selection of new-onset $\mathrm{AF}$ patients, patients with preceding clinical $\mathrm{AF}$ episodes were excluded. To enable us to analyze the $\mathrm{P}$ wave morphology during recent sinus rhythm, patients without a preceding sinus rhythm ECG recording within 12 months were also excluded. Patients prescribed class I or III antiarrhythmic agents were also excluded to eliminate the influence of antiarrhythmic

From the ${ }^{1}$ Department of Cardiovascular Medicine, Kitasato University School of Medicine, Sagamihara, Japan

Address for correspondence: Shinichi Niwano, MD, Department of Cardiovascular Medicine, Kitasato University School of Medicine 1-15-1 Kitasato, Minami-ku, Sagamihara 252-0374, Japan. E-mail: shniwano@med.kitasato-u.ac.jp

Received for publication December 1, 2015. Revised and accepted March 3, 2016.

Released in advance online on J-STAGE September 1, 2016.

All rights reserved by the International Heart Journal Association. 
agents on the $\mathrm{P}$ wave analysis. Finally, 79 patients were enrolled in this study. Patients were classified into PAF and persistent AF patients by the following 1-week observation in accordance with the definitions in the AHA/ACC/ESC and JCS guidelines. $^{7,8)}$ In this study, persistent AF patients had documented AF on an ECG more than 1 week after the first documented AF (without sinus rhythm during this period) when patients visited or were hospitalized. Various clinical parameters, including $\mathrm{P}$ wave morphology, were evaluated in these patients and were compared between the two groups (Figure 1).

For all patients, clinical data, including blood chemistry and echocardiographic data, were evaluated retrospectively from routine examinations of each patient in an outpatient clinic. For the echocardiography (SONOS 7500; Philips, Andover, MA, USA), chamber dimensions were measured in the standard parasternal long-axis view, and the left ventricular ejection fraction was calculated using the area-length method.9 All studies were conducted with the approval of the Ethics Committee of Kitasato University Hospital. Written informed consent was obtained from patients whenever possible, and was received from 32 of 79 patients by retrospective explanation or contact using a letter or fax.

Analysis of the $\mathbf{P}$ wave: For all patients, body-surface 12-lead ECG was recorded (FCP-7541; Fukuda Denshi Co, Ltd, Tokyo) using a standard gain of $0.1 \mathrm{mV} / \mathrm{mm}$ and a recording speed of $25 \mathrm{~mm} / \mathrm{s}$. For the $\mathrm{P}$ wave analysis, the most recent ECG recording of sinus rhythm preceding the new-onset AF episode within 12 months was used. Sinus rhythm was con- firmed from the $\mathrm{P}$ wave axis of $0-90$ degrees in the frontal plane and 0-90 degrees in the horizontal plane. ${ }^{10)}$ In each ECG trace, durations and amplitudes of the $\mathrm{P}$ waves were evaluated in all 12 leads. The onset and offset points of the $\mathrm{P}$ waves were determined as the intersection point of the upward or downward deflection of a $\mathrm{P}$ wave and the isoelectric line. ${ }^{11,12)}$ When the $\mathrm{P}$ waves exhibited biphasic forms, the latter negative phase was also included in the $\mathrm{P}$ wave duration (Figure 2). The $\mathrm{P}$

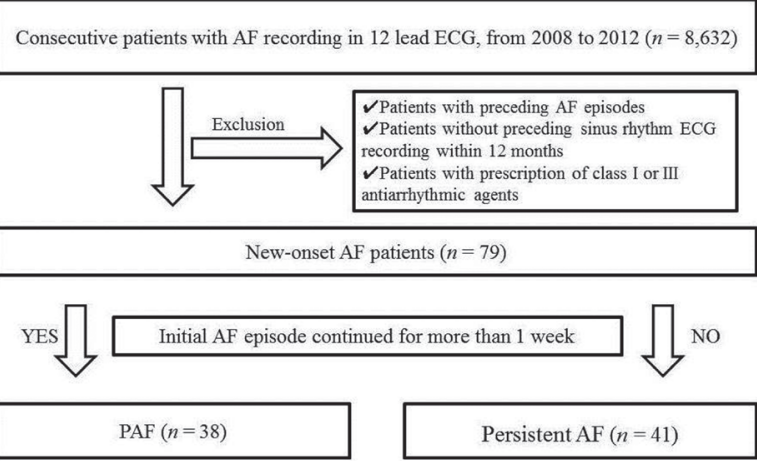

Figure 1. Study population. Of 8,632 AF patients with 12-lead ECG recordings, 79 new-onset AF patients were selected by excluding patients with preceding AF episodes, without preceding sinus rhythm ECG recording within 12 months, or with prescription of class I or III antiarrhythmic agents. Utilizing the following 1 week observation, patients were classified into PAF and persistent AF groups. See text for details.
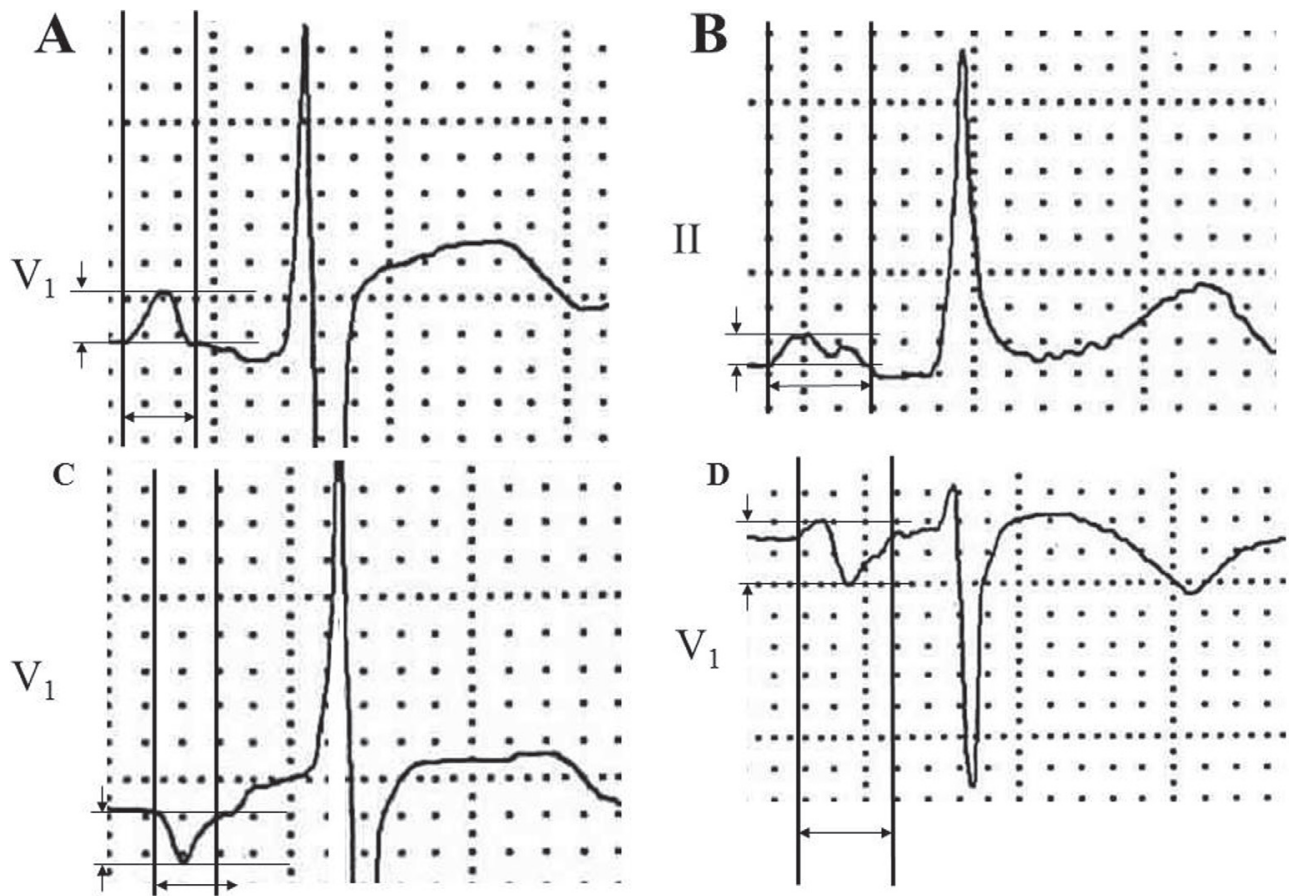

Figure 2. Analysis of $P$ waves. Representative examples of sinus rhythm $P$ wave analysis in a 12-lead ECG recording. The onset and offset points of the $\mathrm{P}$ wave were determined as the intersection point of upward or downward deflection of the $\mathrm{P}$ wave and the isoelectric line (A-C). When a $\mathrm{P}$ wave exhibited a biphasic form, the latter negative phase was also included in the measurement of $\mathrm{P}$ wave duration (D). The $\mathrm{P}$ wave amplitude was measured as the height of the peak of positive deflection or depth of the bottom of negative deflection from the isoelectric line of the onset point (A-C). In the biphasic P wave, the $\mathrm{P}$ wave amplitude was measured as the difference between the positive peak and the negative bottom of the recording (D). See text for details. 
Table I. Clinical Characteristics of Patients and Univariate Analysis

\begin{tabular}{|c|c|c|c|c|}
\hline & $\begin{array}{c}\text { Total } \\
n=79\end{array}$ & $\begin{array}{c}\text { PAF } \\
n=38\end{array}$ & $\begin{array}{l}\text { Persistent AF } \\
\quad n=41\end{array}$ & $P$ \\
\hline Age (years) & $70 \pm 11$ & $68 \pm 12$ & $72 \pm 10$ & 0.124 \\
\hline Gender (M:F) & $46: 33$ & $23: 15$ & $23: 18$ & 0.690 \\
\hline BMI $\left(\mathrm{kg} / \mathrm{m}^{2}\right)$ & $21.7 \pm 10.8$ & $21.8 \pm 3.9$ & $21.7 \pm 3.7$ & 0.970 \\
\hline Smoking $n(\%)$ & $38(50.7)$ & $17(22.7)$ & $21(28.0)$ & 0.570 \\
\hline \multicolumn{5}{|l|}{ Underlying disease } \\
\hline Congestive heart failure $n(\%)$ & $25(31.7)$ & $6(7.6)$ & $19(24.1)$ & $0.004^{*}$ \\
\hline Ischemic heart disease $n(\%)$ & $23(29.2)$ & $10(12.7)$ & $13(16.5)$ & 0.612 \\
\hline Dilated cardiomyopathy $n(\%)$ & $1(1.3)$ & $0(0.0)$ & $1(1.3)$ & 0.342 \\
\hline Hypertrophic cardiomyopathy $n(\%)$ & $3(3.8)$ & $2(2.5)$ & $1(1.3)$ & 0.514 \\
\hline Left ventricular hypertrophy $n(\%)$ & $13(16.5)$ & $4(5.1)$ & $9(11.4)$ & 0.174 \\
\hline Valvular heart disease $n(\%)$ & $6(7.6)$ & $0(0.0)$ & $6(7.6)$ & $0.014^{*}$ \\
\hline Hypertension $n(\%)$ & $58(73.4)$ & $23(29.1)$ & $35(44.3)$ & $0.013^{*}$ \\
\hline Diabetes mellitus $n(\%)$ & $25(31.6)$ & $8(10.1)$ & $17(21.5)$ & 0.051 \\
\hline Hyperlipidemia $n(\%)$ & $38(48.1)$ & $12(15.2)$ & $26(32.9)$ & $0.005^{*}$ \\
\hline Vascular disease $n(\%)$ & $7(8.9)$ & $1(1.3)$ & $6(7.6)$ & 0.061 \\
\hline \multicolumn{5}{|l|}{ ECG parameters } \\
\hline Heart rate (sinus) (bpm) & $69 \pm 14$ & $70 \pm 16$ & $67 \pm 13$ & 0.402 \\
\hline Heart rate $(\mathrm{AF})(\mathrm{bpm})$ & $98 \pm 28$ & $103 \pm 30$ & $93 \pm 24$ & 0.114 \\
\hline Maximum $\mathrm{P}$ wave duration (msec) & $106.6 \pm 15.5$ & $103.4 \pm 16.9$ & $109.5 \pm 13.6$ & 0.082 \\
\hline $\mathrm{P}$ wave dispersion (msec) & $61.7 \pm 14.6$ & $56.6 \pm 14.8$ & $66.5 \pm 12.8$ & $0.002^{*}$ \\
\hline P wave amplitude in II (mV) & $0.165 \pm 0.055$ & $0.170 \pm 0.009$ & $0.160 \pm 0.009$ & 0.424 \\
\hline $\mathrm{P}$ wave amplitude in $\mathrm{V}_{1}(\mathrm{mV})$ & $0.149 \pm 0.084$ & $0.149 \pm 0.014$ & $0.149 \pm 0.013$ & 1.000 \\
\hline \multicolumn{5}{|l|}{ UCG parameters } \\
\hline Left atrial dimension (mm) & $44 \pm 9$ & $40 \pm 7$ & $48 \pm 8$ & $<0.0001^{*}$ \\
\hline Left ventricular ejection fraction $(\%)$ & $62 \pm 10$ & $61 \pm 10$ & $63 \pm 9$ & 0.440 \\
\hline Left ventricular diastolic dimension (mm) & $50 \pm 7$ & $48 \pm 6$ & $52 \pm 8$ & 0.021 \\
\hline \multicolumn{5}{|l|}{ Blood chemistry } \\
\hline $\mathrm{BNP}(\mathrm{pg} / \mathrm{mL})$ & $226.8 \pm 374.6$ & $205.2 \pm 269.6$ & $244.5 \pm 445.1$ & 0.670 \\
\hline $\mathrm{Cr}(\mathrm{mg} / \mathrm{dL})$ & $1.03 \pm 0.76$ & $1.01 \pm 0.79$ & $1.05 \pm 0.12$ & 0.804 \\
\hline eGFR (mL/minute) & $64.3 \pm 25.7$ & $66.6 \pm 27.4$ & $62.3 \pm 24.1$ & 0.460 \\
\hline $\operatorname{HbA} 1 \mathrm{c}(\%)$ & $5.4 \pm 2.1$ & $4.8 \pm 2.5$ & $6.0 \pm 1.3$ & $0.004^{*}$ \\
\hline LDL cholesterol (mg/dL) & $106.9 \pm 34.8$ & $102.3 \pm 35.2$ & $110.3 \pm 34.6$ & 0.362 \\
\hline $\mathrm{TG}(\mathrm{mg} / \mathrm{dL})$ & $128.8 \pm 74.1$ & $142.2 \pm 90.6$ & $117.8 \pm 56.1$ & 0.173 \\
\hline \multicolumn{5}{|l|}{ Prescription } \\
\hline $\mathrm{ARB} / \mathrm{ACEI} n(\%)$ & $37(46.9)$ & $13(16.5)$ & $24(30.4)$ & $0.031^{*}$ \\
\hline Spironolactone $n(\%)$ & $8(10.2)$ & $1(1.3)$ & $7(8.9)$ & $0.034^{*}$ \\
\hline$\beta$-blocker $n(\%)$ & $31(39.3)$ & $10(12.7)$ & $21(26.6)$ & $0.024^{*}$ \\
\hline Ca-blocker $n(\%)$ & $28(35.4)$ & $11(13.9)$ & $17(11.5)$ & 0.244 \\
\hline Statin $n(\%)$ & $25(31.6)$ & $8(10.1)$ & $17(21.5)$ & 0.051 \\
\hline
\end{tabular}

BMI indicates body mass index; BNP, brain natriuretic peptide; $\mathrm{Cr}$, creatinine; eGFR, estimated glomerular filtration rate; LDL cholesterol, low-density lipoprotein cholesterol; TG, triacylglycerol; ARB, angiotensin receptor blocker; and ACEI, angiotensin converting enzyme inhibitor. ${ }^{*}$ Statistical significance.

wave amplitude was measured as the height of the peak of positive deflection or the depth of the bottom of negative deflection from the isoelectric line of the onset point. In the biphasic $\mathrm{P}$ waves, the $\mathrm{P}$ wave amplitude was measured as the difference between the positive peak and the negative bottom of the recording (Figure 2). In the present study, positive deflections of $\mathrm{P}$ waves were also included into the analysis to salvage the electrophysiological properties of the right atria. ${ }^{13)}$ When there was difficulty in the determination of the onset or offset points of the $\mathrm{P}$ wave due to low $\mathrm{P}$ wave amplitude, data from the associated lead was excluded from the analysis. The digitally-stored ECG signals with time and amplitude resolutions of $1 \mathrm{msec}$ and $0.001 \mathrm{mV}$, respectively, (FCP-7541; Fukuda Denshi Co, Ltd, Tokyo) were used for P wave evaluation. The analyses were performed by 2 investigators blinded to the other clinical parameters, and the means of their measurements were used. When the difference between the 2 investigators exceeded $12 \mathrm{msec}$ in duration or $0.03 \mathrm{mV}$ in amplitude, onset- offset or peak-bottom points were discussed between the 2 investigators for the data adjustment. For the $\mathrm{P}$ wave analysis, durations and amplitudes of the P waves in leads II and V1 and the maximum $\mathrm{P}$ wave duration in the 12 leads were used as analysis parameters. ${ }^{14-16)}$ In addition, the dispersion of the $\mathrm{P}$ wave duration ( $\mathrm{P}$ wave dispersion) was calculated by subtracting the minimum $\mathrm{P}$ wave duration from the maximum $\mathrm{P}$ wave duration among the 12 leads. ${ }^{11,12,14-17)}$

Statistical analysis: All clinical data were compared between the PAF and persistent AF groups by univariate analysis to determine useful indices for discriminating between PAF and persistent AF patients. Multivariate analysis was performed using the parameters that showed a $P$ value of $<0.1$ in the univariate analysis by a stepwise method to identify the independent factors that predict persistent AF patients. This parameter selection was employed to eliminate various correlated parameters in our relatively small sized study population. For the independent predictive factors, receiver-operating characteristic 
Table II. Multivariate Analysis of Clinical Parameters for Discrimination Between Persistent AF and PAF Patients

\begin{tabular}{lccc}
\hline & Odds ratio & $95 \%$ CI & $P$ \\
\hline Maximum P wave duration (msec) & 0.94 & $0.87-1.00$ & 0.0511 \\
P wave dispersion (msec) & 1.11 & $1.04-1.21$ & $0.0014^{*}$ \\
Left atrial dimension (mm) & 1.15 & $1.06-1.27$ & $0.0003^{*}$ \\
HbAlc (\%) & 1.53 & $0.92-2.33$ & 0.0644 \\
\hline
\end{tabular}

"Statistical significance.
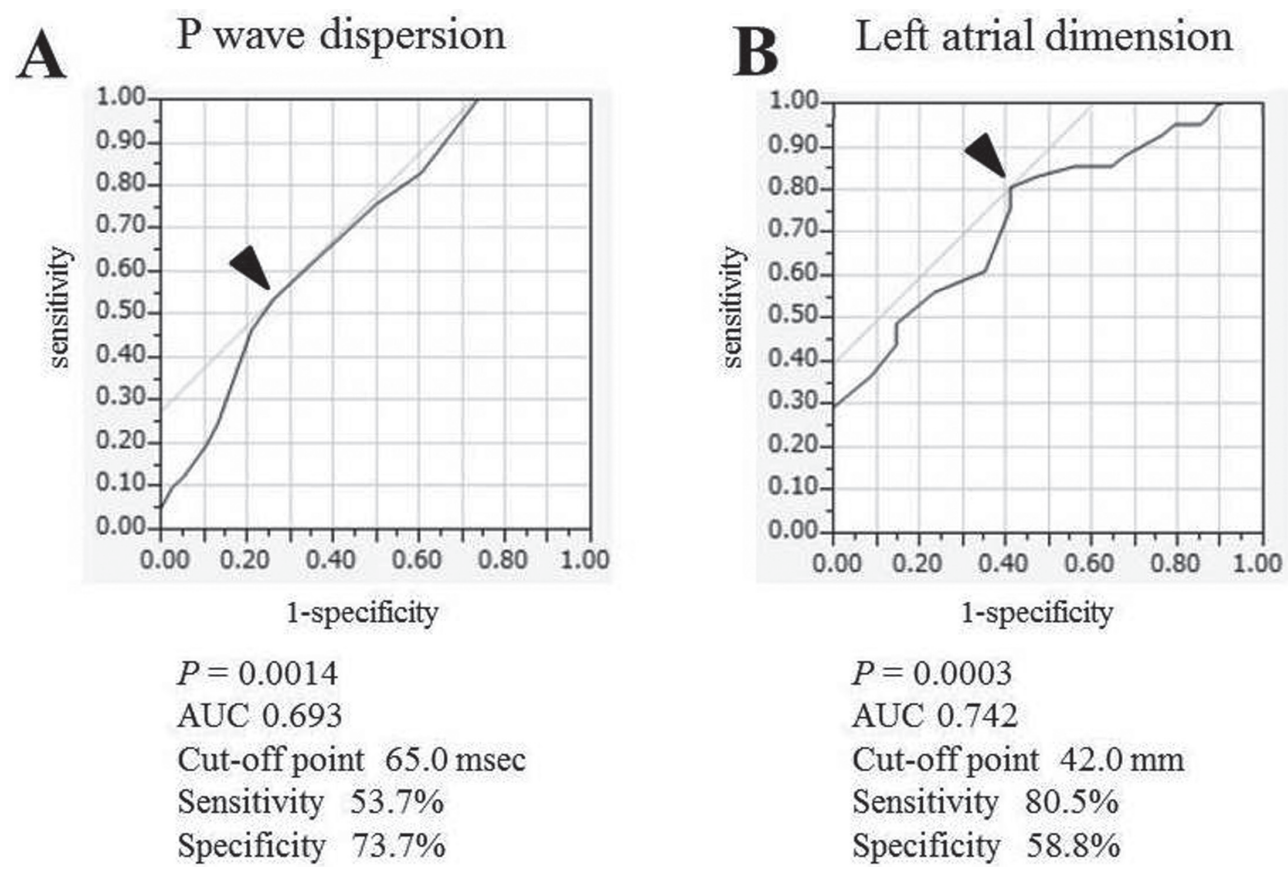

Figure 3. ROC curves for determination of PAF and persistent AF patients. ROC curves of $P$ wave dispersion and LAD for determination of persistent AF patients in logistic regression analysis. The arrows indicate the optimal cut-off points for determination of PAF and persistent AF patients in each parameter. $\mathrm{P}$ wave dispersion achieved a sensitivity of $53.7 \%$ and specificity of $73.7 \%$ by setting the cut-off point at $\mathrm{P}$ wave dispersion $>65.0 \mathrm{msec}(P=0.0014$, area under curve [AUC] 0.693 , A). Left atrial dimension achieved a sensitivity of $80.5 \%$ and specificity of $58.8 \%$ by setting the cut-off point at left atrial dimension $>42.0 \mathrm{~mm}(P=0.0003$, AUC $0.742, \mathbf{B})$. See text for details.

(ROC) curves were obtained by logistic regression analysis. The optimal cutoff point was determined to achieve the highest sensitivity and specificity for each ROC curve for the determination of persistent AF patients. Finally, the c-statistics using significant parameters were calculated.

The JMP 10 (SAS Japan Inc., Tokyo) statistical software package was used for statistical analysis. Student's $t$-test or the Mann-Whitney $U$ test was used to compare continuous variables, which are presented as the mean \pm standard deviation. The $\chi^{2}$ test was used to compare dichotomous variables, which are presented as percentages. Logistic regression analysis was performed to obtain ROC curves. A Cox hazard model was used for multivariate analysis. $P$ values of $<0.05$ were considered to indicate statistical significance.

\section{RESUltS}

Clinical characteristics of patients and univariate analysis: Clinical profiles and characteristics of the study population are summarized in Table I. The incidence of underlying disease was higher in persistent AF patients than in PAF patients, and significant differences in the presence of congestive heart failure, valvular heart disease, hypertension, and hyperlipidemia were observed between the groups. $\mathrm{P}$ wave dispersion $(P=$ 0.002), left atrial dimension (LAD) $(P<0.0001)$, and left ventricular diastolic dimension $(P=0.021)$ were significantly larger in persistent AF patients compared with PAF patients. Hemoglobin A1c (HbA1c) levels were higher in persistent AF patients $(P=0.004)$. Prescription of various cardiovascular-active medicines was also more frequent in persistent $\mathrm{AF}$ patients than in PAF patients (Table I).

Multivariate analysis: For the multivariate analysis, parameters were selected by the stepwise method from those which exhibited $P<0.1$ in the univariate analysis to avoid inappropriate repeated selection of parameters in similar categories. As a result, maximum $\mathrm{P}$ wave duration, $\mathrm{P}$ wave dispersion, $\mathrm{LAD}$, and hemoglobin A1c were selected, and $\mathrm{P}$ wave dispersion and LAD were identified as independent factors for discrimination between PAF and persistent AF patients (Table II).

Discrimination between PAF and persistent AF: Figure 3 shows the ROC curves of $\mathrm{P}$ wave dispersion and LAD for de- 
Table III. Discrimination of Patients by P wave Dispersion and LAD Diagnosis as Non- PAF Patients

\begin{tabular}{lcccc}
\hline & & & PWD $>65 \mathrm{msec}$ & PWD $>65 \mathrm{msec}$ \\
or & and & LAD $>42 \mathrm{~mm}$ & LAD $>42 \mathrm{~mm}$ \\
\hline Sensitivity & & LAD $>42 \mathrm{~mm}$ & 0.419 & 0.953 \\
Specificity & 0.537 & 0.805 & 0.889 & 0.444 \\
Positive predictive value & 0.737 & 0.588 & 0.818 & 0.672 \\
\hline
\end{tabular}

PWD indicates $\mathrm{P}$ wave dispersion.

termination of persistent $\mathrm{AF}$ patients in the logistic regression analysis. The most significant cut-off point was $>65.0 \mathrm{msec}$ for $\mathrm{P}$ wave dispersion, with a sensitivity of $53.7 \%$ and a specificity of $73.7 \%$, and $>42.0 \mathrm{~mm}$ for LAD, with a sensitivity of $80.5 \%$ and a specificity of $58.8 \%$. Table III shows the results using $\mathrm{P}$ wave dispersion of $>65 \mathrm{msec}$ and/or LAD of $>42 \mathrm{~mm}$ as the criteria for discrimination between PAF and persistent AF patients, which together achieved a specificity of $88.9 \%$ and a positive predictive value of $81.8 \%$. In contrast, use of $\mathrm{P}$ wave dispersion of $>65 \mathrm{msec}$ or LAD of $>42 \mathrm{~mm}$ achieved a sensitivity of $95.3 \%$ and a negative predictive value of $88.9 \%$. The c-statistics of predicting persistent AF improved from $0.742,0.693$ to 0.799 by adding $\mathrm{P}$ wave dispersion to LAD.

\section{Discussion}

The present study evaluated the differences in various clinical parameters between PAF and persistent AF patients, demonstrated with several interesting findings. First, several clinical parameters, including underlying diseases, $\mathrm{P}$ wave dispersion, LAD, HbAlc level, and the prescription of various cardiovascular-active medicines, showed significant differences between PAF and persistent AF patients in the univariate analysis. Second, $\mathrm{P}$ wave dispersion and LAD were identified as independent factors for discrimination between PAF and persistent AF patients in the multivariate analysis. Finally, when the optimal cut-off points of these two parameters were used as criteria for discrimination between PAF and persistent AF patients, the combination of the two achieved relatively high sensitivity (88.9\%) and specificity $(95.3 \%)$.

Parameters to discriminate between PAF and persistent AF: Several studies have reported the importance of $\mathrm{P}$ wave dispersion as a marker of the clinical phase of $\mathrm{AF}{ }^{18-20)}$ suggesting that $\mathrm{P}$ wave dispersion may be prolonged with progression of the AF phase, ie, the change from the PAF to persistent AF phase. Furthermore, Koide, et al concluded that $\mathrm{P}$ wave dispersion measurement may be useful in determining the progression of the clinical phase of $\mathrm{AF}^{19)}$ In the present study, multivariate analysis identified $\mathrm{P}$ wave dispersion as an independent factor for discrimination between PAF and persistent AF patients, consistent with previous reports. The mechanism of such significance of $\mathrm{P}$ wave dispersion is unclear. In the consideration of single and same $\mathrm{P}$ wave vector in the ECG recording, some investigators have doubted the clinical usefulness of this dispersion, but our present and preceding studies and other investigators have stated that the $\mathrm{P}$ wave dispersion is a useful index with which to evaluate atrial electrophysiology. ${ }^{6,11,12,14-16,19)}$ Because $\mathrm{P}$ wave dispersion is the relative parameter calculated from the other measurements, correction or ad- aptation of direct value by $\mathrm{P}$ wave duration or heart rate might be a possible method to obtain a parameter with absolute meaning, but because we could not find such a trial about correction in dispersion in the previous reports, we just used direct data of dispersion in this study. The mechanism underlying $\mathrm{P}$ wave dispersion change remains uncertain, but may partially be explained by the change in direction of the atrial activation vector. The atrial activation time is speculated to be prolonged along with the progression of $\mathrm{AF}$, due to the atrial enlargement and tissue degeneration as a result of atrial structural remodeling in AF patients. ${ }^{14,21,22)}$ Several studies have suggested that these changes may directly result in the prolongation of $\mathrm{P}$ wave duration, ${ }^{23-25)}$ but may also influence the atrial activation vector. In particular, the terminal portion of the atrial activation vector may be generated by a smaller amount of atrial tissue, resulting in a smaller amplitude signal. This activation may be recorded in some leads, but is likely to not be detected in leads with a horizontal angle to the direction of vector. ${ }^{6,16,26)}$ This phenomenon may result in the pseudo-disappearance of the terminal portion of the $\mathrm{P}$ wave in some leads, resulting in the enlargement of $\mathrm{P}$ wave dispersion in all 12 leads. In the present study, $\mathrm{P}$ wave duration also tended to be longer in persistent AF patients than in PAF patients, but the difference was not significant. In contrast, $\mathrm{P}$ wave dispersion exhibited a significant difference between the two groups, indicating that $\mathrm{P}$ wave dispersion was a more sensitive parameter in discriminating the clinical phase of $\mathrm{AF}$ in the present study population.

The present results also suggested LAD may be another parameter for discrimination between PAF and persistent AF patients, consistent with previous studies reporting an association between LAD and AF clinical history. ${ }^{18,27-29)}$ However, the underlying cause of LA enlargement may be more complicated than the above explanation. Because we selected "new-onset AF" patients in the present study, LA enlargement may not only be due to AF, but may also be influenced by other underlying conditions. Although underlying diseases were not selected in the multivariate analysis using the stepwise method, univariate analysis showed higher prevalence of congestive heart failure, valvular heart disease, and hypertension in persistent AF patients, suggesting such underlying diseases may support atrial conditions more suitable for AF persistence, even before the clinical onset of AF.

Combined use of two parameters to discriminate between PAF and persistent AF: Koide, et al reported that the progression of PAF to persistent AF can be predicted by $\mathrm{P}$ wave dispersion, with a sensitivity of $71 \%$, specificity of $77 \%$, positive predictive value of $63 \%$, and negative predictive value of $83 \%{ }^{19)}$ Similarly, they reported that such AF progression can be detected by LA enlargement with a sensitivity of $64 \%$, specificity of $76 \%$, positive predictive value of $59 \%$, and negative predic- 
tive value of $79 \% .^{18)}$ In the present study, although we identified $\mathrm{P}$ wave dispersion and LAD as independent predictive factors for discrimination between PAF and persistent AF patients, the sensitivity and specificity of each parameter for PAF and/or persistent AF discrimination were limited (Table III). However, combination of both parameters achieved very high sensitivity and specificity, which may be useful for distinguishing PAF from persistent AF patients in clinical practice.

Clinical implications: The efficacy of rhythm control therapy is much higher in PAF patients than in persistent AF patients, regardless of a pharmacological or non-pharmacological, ie, catheter ablation, approach. Thus, a diagnosis of PAF strongly supports the choice of rhythm control therapy in clinical practice. ${ }^{1,30)}$ This discrimination also enables evaluation of catheter ablation efficacy as well as the necessity of additional procedures for standard pulmonary vein isolation, ie, linear ablation, continuous fractionated atrial electrogram ablation, and/or ganglionated plexi ablation. ${ }^{1,431,32)}$ Additionally, because the discrimination of PAF and persistent AF patients in the present study population was in "new-onset AF" patients, the present results are applicable for "first documented AF" patients. To the best of our knowledge, this is the first report which stated the parameters to discriminate between $\mathrm{PAF}$ and persistent $\mathrm{AF}$ patients in cases with "new-onset" AF. The clinical phase of $\mathrm{AF}$ is important in considering therapeutic strategy in AF patients; however, determination of the phase takes time, because it is usually accomplished after clinical observation. ${ }^{33)}$ The present study has identified factors to discriminate the clinical phase of AF, including in "first documented AF" patients, which may be useful for choosing the appropriate therapeutic strategy in patients. Although several preceding manuscripts analyzing $\mathrm{P}$ wave and $\mathrm{P}$ wave dispersion in $\mathrm{AF}$ cases can be found, discrimination of PAF and persistent AF cases in 'newonset' AF patients is a completely novel trial.

Limitations: The present study included several limitations. First, the size of the study population was relatively small, so that some parameters might be too enhanced in the analysis because of some patients with deviated data. Second, since the time resolution of screening AF in ECG recordings was too inaccurate, we might have failed to document "preceding" AF episodes even in our patient population of "new-onset AF". This is the systematic limitation of this study. Third, because the measurement of $\mathrm{P}$ waves was based on morphological characterization, measurement errors may have been included to some degree. Finally, although the predictive factors for the clinical stage of AF in the present study showed good sensitivity and specificity, they were only evaluated in a limited study population, in which the predictive factors themselves had been determined by multivariate analysis. These issues should be resolved in a future prospective study with a larger patient population.

Conclusions: In patients with new-onset AF, $\mathrm{P}$ wave dispersion and LAD were independent factors for discrimination between PAF and persistent AF patients in multivariate analysis. Combination of both factors achieved high sensitivity, specificity, positive prediction value, and negative prediction value. These factors may be useful for the determination of clinical strategies in new-onset AF patients.

\section{Disclosure}

This study received no financial support from commercial sources, and the authors state no conflicts of interest. There was no unapproved use of any compound or product.

\section{REFERENCES}

1. Hakalahti A, Biancari F, Nielsen JC, Raatikainen MJ. Radiofrequency ablation vs. antiarrhythmic drug therapy as first line treatment of symptomatic atrial fibrillation: systematic review and meta-analysis. Europace 2015; 17: 370-8. (Review)

2. Wilber DJ, Pappone C, Neuzil P, et al. Comparison of antiarrhythmic drug therapy and radiofrequency catheter ablation in patients with paroxysmal atrial fibrillation: a randomized controlled trial. JAMA 2010; 303: 333-40.

3. Mont L, Bisbal F, Hernández-Madrid A, et al. Catheter ablation vs. antiarrhythmic drug treatment of persistent atrial fibrillation: a multicentre, randomized, controlled trial (SARA study). Eur Heart J 2014; 35: 501-7.

4. Wynn GJ, Das M, Bonnett LJ, Panikker S, Wong T, Gupta D. Efficacy of catheter ablation for persistent atrial fibrillation: a systematic review and meta-analysis of evidence from randomized and nonrandomized controlled trials. Circ Arrhythm Electrophysiol 2014; 7: 841-52. (Review)

5. Haegeli LM, Calkins H. Catheter ablation of atrial fibrillation: an update. Eur Heart J 2014; 35: 2454-9. (Review)

6. Yoshizawa T, Niwano S, Niwano $\mathrm{H}$, et al. Prediction of new onset atrial fibrillation through $\mathrm{P}$ wave analysis in 12 lead ECG. Int Heart J 2014; 55: 422-7.

7. Fuster V, Rydén LE, Cannom DS, et al. ACC/AHA/ESC 2006 Guidelines for the Management of Patients with Atrial Fibrillation: a report of the American College of Cardiology/American Heart Association Task Force on Practice Guidelines and the European Society of Cardiology Committee for Practice Guidelines (Writing Committee to Revise the 2001 Guidelines for the Management of Patients With Atrial Fibrillation): developed in collaboration with the European Heart Rhythm Association and the Heart Rhythm Society. Circulation 2006; 114: e257-354.

8. JCS Joint Working Group. Guidelines for Pharmacotherapy of Atrial Fibrillation (JCS 2013). Circ J 2014; 78: 1997-2021.

9. Lang RM, Badano LP, Mor-Avi V, et al. Recommendations for cardiac chamber quantification by echocardiography in adults: an update from the American Society of Echocardiography and the European Association of Cardiovascular Imaging. J Am Soc Echocardiogr 2015; 28: 1-39.

10. Blomström-Lundqvist C, Scheinman MM, Aliot EM, et al. ACC/ AHA/ESC guidelines for the management of patients with supraventricular arrhythmias--executive summary. a report of the American college of cardiology/American heart association task force on practice guidelines and the European society of cardiology committee for practice guidelines (writing committee to develop guidelines for the management of patients with supraventricular arrhythmias) developed in collaboration with NASPE-Heart Rhythm Society. J Am Coll Cardiol 2003; 42: 1493-531. (Review)

11. Dogan A, Avsar A, Ozturk M. P-wave dispersion for predicting maintenance of sinus rhythm after cardioversion of atrial fibrillation. Am J Cardiol 2004; 93: 368-71.

12. Karaca I, Durukan P, Dagli N, Yavuskir M, Ikizceli I, Balin M. The effect of rapid blood pressure control on P-wave dispersion in hypertensive urgency. Adv Ther 2008; 25: 1303-14.

13. Baranchuk A, Bayés de Luna A. The P-wave morphology: what does it tell us? Herzschrittmacherther Elektrophysiol 2015; 26: 192-9.

14. Wong T, Davlouros PA, Li W, Millington-Sanders C, Francis DP, Gatzoulis MA. Mechano-electrical interaction late after Fontan operation: relation between $\mathrm{P}$-wave duration and dispersion, right atrial size, and atrial arrhythmias. Circulation 2004; 109: 2319-25. 
15. Yamada T, Fukunami M, Shimonagata T, et al. Dispersion of signal-averaged $\mathrm{P}$ wave duration on precordial body surface in patients with paroxysmal atrial fibrillation. Eur Heart J 1999; 20: 211-20.

16. Tsioufis C, Syrseloudis D, Hatziyianni A, et al. Relationships of $\mathrm{CRP}$ and $\mathrm{P}$ wave dispersion with atrial fibrillation in hypertensive subjects. Am J Hypertens 2010; 23: 202-7.

17. Akutsu Y, Kaneko K, Kodama Y, et al. A combination of $\mathrm{P}$ wave electrocardiography and plasma brain natriuretic peptide level for predicting the progression to persistent atrial fibrillation: comparisons of sympathetic activity and left atrial size. J Interv Card Electrophysiol 2013; 38: 79-84.

18. Koide Y, Yotsukura M, Sakata K, Yoshino H, Ishikawa K. Investigation of the predictors of transition to persistent atrial fibrillation in patients with paroxysmal atrial fibrillation. Clin Cardiol 2002; 25: 69-75.

19. Koide Y, Yotsukura M, Ando $\mathrm{H}$, et al. Usefulness of $\mathrm{P}$ wave dispersion in standard twelve-lead electrocardiography to predict transition from paroxysmal to persistent atrial fibrillation. Am J Cardiol 2008; 102: 573-7.

20. Akutsu Y, Kaneko K, Kodama Y, et al. A combination of P wave electrocardiography and plasma brain natriuretic peptide level or predicting the progression to persistent atrial fibrillation: comparisons of sympathetic activity and left atrial size. J Interv Card Electrophysiol 2013; 38: 79-84.

21. Morton JB, Sanders P, Vohra JK, et al. Effect of chronic right atrial stretch on atrial electrical remodeling in patients with an atrial septal defect. Circulation 2003; 107: 1775-82

22. Thilén U, Carlson J, Platonov PG, Havmöller R, Olsson SB. Prolonged $\mathrm{P}$ wave duration in adults with secundum atrial septal defect: a marker of delayed conduction rather than increased atrial size? Europace 2007; 9: vi105-8.

23. Savarino P, Borgarelli M, Tarducci A, Crosara S, Bello NM, Margiocco ML. Diagnostic performance of $\mathrm{P}$ wave duration in the identification of left atrial enlargement in dogs. J Small Anim Pract 2012; 53: 267-72
24. Zeng C, Wei T, Zhao R, Wang C, Chen L, Wang L. Electrocardiographic diagnosis of left atrial enlargement in patients with mitral stenosis: the value of the P-wave area. Acta Cardiol 2003; 58: 13941.

25. Birkbeck JP, Wilson DB, Hall MA, Meyers DG. P-wave morphology correlation with left atrial volumes assessed by 2-dimensional echocardiography. J Electrocardiol 2006; 39: 225-9.

26. Acar RD, Bulut M, Ergün S, Yesin M, Boztosun B, Akçakoyun M. $\mathrm{P}$-wave dispersion and its relationship to aortic stiffness in patient with acute myocardial infarction after cardiac rehabilitation. ARYA Atheroscler 2014; 10: 185-91.

27. Abe Y, Fukunami M, Yamada T, Ohmori M, et al. Prediction of transition to chronic atrial fibrillation in patients with paroxysmal atrial fibrillation by signal-averaged electrocardiography: a prospective study. Circulation 1997; 96: 2612-6.

28. Sakamoto H, Kurabayashi M, Nagai R, Fujii J. Prediction of transition to chronic atrial fibrillation in patients with paroxysmal atrial fibrillation. Circulation 1998; 98: 1045-6.

29. Parkash R, Green MS, Kerr CR, et al. The association of left atrial size and occurrence of atrial fibrillation: a prospective cohort study from the Canadian Registry of Atrial Fibrillation. Am Heart J 2004; 148: 649-54

30. Komatsu T, Yomogida K, Nakamura S, et al. Relationship between duration of arrhythmia and subsequent preventive effect of disopyramide after cardioversion in patients with symptomatic paroxysmal and persistent atrial fibrillation. J Cardiol 2003; 42: 1117. (Japanese)

31. Romero J, Gianni C, Di Biase L, Natale A. Catheter Ablation for Long-Standing Persistent Atrial Fibrillation. Methodist Debakey Cardiovasc J 2015; 11: 87-93. (Review)

32. Willems S, Klemm H, Rostock T, et al. Substrate modification combined with pulmonary vein isolation improves outcome of catheter ablation in patients with persistent atrial fibrillation: a prospective randomized comparison. Eur Heart J 2006; 27: 2871-8.

33. JCS Joint Working Group. Guidelines for Pharmacotherapy of Atrial Fibrillation (JCS 2013). Circ J 2014; 78: 1997-2021. 